$G A-A-20876$

DE92 012699

\title{
A PHASE CONTRAST INTERFEROMETER ON DIII-D
}

by 


\section{DISCLAIMER}

This report was prepared as an account of work sponsored by an agency of the United States Government. Neither the United States Government nor any agency thereof, nor any of their employees, makes any warranty, express or implied, or assuines any legal liability or responsibility for the accuracy, completeness, or usefulness of any information, apparatus, product, or process disclosed, or represents that its use would not infringe privately owned rights. Reference herein to any specific commercial product, process, or service by trade name, trademark, manufacturer, or otherwise, does not necessarily constitute or imply its endorsement, recommendation, or favoring by the United States Government or any agency thereof. The views and opinions of authors expressed herein do not necessarily state or reflect those of the United States Government or any agency thereof. 
GA-A20876

\title{
A PHASE CONTRAST INTERFEROMETER ON DIII-D
}

\author{
by \\ S. CODA, ${ }^{*}$ M. PORKOLAB, ${ }^{\dagger}$ T.N. CARLSTROM \\ *MIT, present address: General Atomics \\ $t_{\text {Massachusetts Institute of Technology (MIT) }}$
}

This is a preprint of a paper to be presented at the 9th Topical Conference on High-Temperature Plasma Diagnostics, March 16-20, 1992, Santa Fe, New Mexico and to be printed in the Proceedings.

\author{
Work supported by \\ Department of Energy \\ Contract DE-AC03-89ER51114
}
GENERAL ATOMICS PROJECT 3466
APRIL 1992

\section{GENERAL}

ATOMICS 


\begin{abstract}
A novel imaging diagnostic has recently become operational on the DIII-D tokamak for the study of density fluctuations at the outer edge of the plasma. The phase contrast imaging approach overcomes the limitations of conventional scattering techniques in the spectral range of interest for transport-related phenomena, by allowing detection of long wavelength modes (up to $7.6 \mathrm{~cm}$ ) with excellent spatial resolution $(5 \mathrm{~mm})$ in the radial direction. Additional motivation for the diagnostic is provided by wave-plasma interactions during heating and current drive experiments in the Ion Cyclotron range of frequencies. Density perturbations of $4 \times 10^{7} \mathrm{~cm}^{-3}$ with a $1 \mathrm{MHz}$ bandwidth can be resolved. The diagnostic employs a $7.6 \mathrm{~cm}$ diameter $\mathrm{CO}_{2}$ laser beam launched vertically across the plasma edge. An image of the plasma is then created on a 16-element detector array: the detector signals are directly proportional to the density fluctuations integrated along each chord. Wavelengths and correlation lengths can be inferred from the spatial mapping. The phase contrast method and its application to DIII-D are described and tests and first plasma data are presented.
\end{abstract}




\section{INTRODUCTION}

Recent experimental ${ }^{1}$ and theoretical ${ }^{2}$ work have indicated that turbulent derisity fluctuations at the edge of tokamak plasmas may be dominated by low wavenumber modes, with wavelengths of the order of a few to tens of ion Larmor radii, and that these modes are chiefly responsible for anomalous transport. ${ }^{3}$ Conventional and widely used diagnostics for the measurement of density fluctuations, such as $\mathrm{CO}_{2}{ }^{4}$ or far infrared ${ }^{5}$ scattering, suffer from lack of spatial resolution at such wavelengths; microwave reflectometry measurements thus far have lacked wavenumber resolution. ${ }^{6}$ The direct spatial representation provided by imaging methods is more attractive at the low wavenumbers, where the scale lengths of the modes become comparable to the characteristic gradient lengths. An imaging diagnostic is also an ideal tool for studying the physics of plasma interaction with if waves during ICH or lower hybrid experiments: our goal during the fast wave current drive experiments ${ }^{7}$ will be to investigate primary waves as well as parametric decay activity. ${ }^{8}$

The phase contrast method is an ideal choice among imaging techniques, since it uniquely combines high sensitivity with a response function which is approximately independent of wavenumber above a given cutof $k_{c}$. To our knowledge, this technique which is well known in the microscopy field ${ }^{0}$ has been applied previously only to two high-density plasma experiments. ${ }^{10,11}$ In our experiment, we use a $\mathrm{CO}_{2}$ laser beam $\left(\lambda_{0}=10.6 \mu \mathrm{m}\right)$ with a diameter of $7.6 \mathrm{~cm}$, which is launched vertically through the outer edge of the DIII-D plasma (see Fig. 1). The detector is a 16 -element, $\mathrm{LN}_{2}$-cooled, $\mathrm{HgMnTe}$ linear photovoltaic array, with a bandwidth of $100 \mathrm{MHz}$. Low intrinsic detector noise insures that a phase serisitivity of $\simeq 10^{-6}$ radians can be attained, corresponding to a minimum detectable density of approximately $4 \times 10^{7} \mathrm{~cm}^{-3}$.

\section{TRANSMISSION TECHNIQUES AND PH CE CONTRAST}

The laser beam experiences a phase shift upon traversing the plasma equal to ${ }^{12}$ (neglecting any toroidal dependence)

$$
\Delta \Phi(R, t) \simeq r_{\mathrm{e}} \lambda_{0} \int n_{\mathrm{e}}(R, z, t) d z
$$




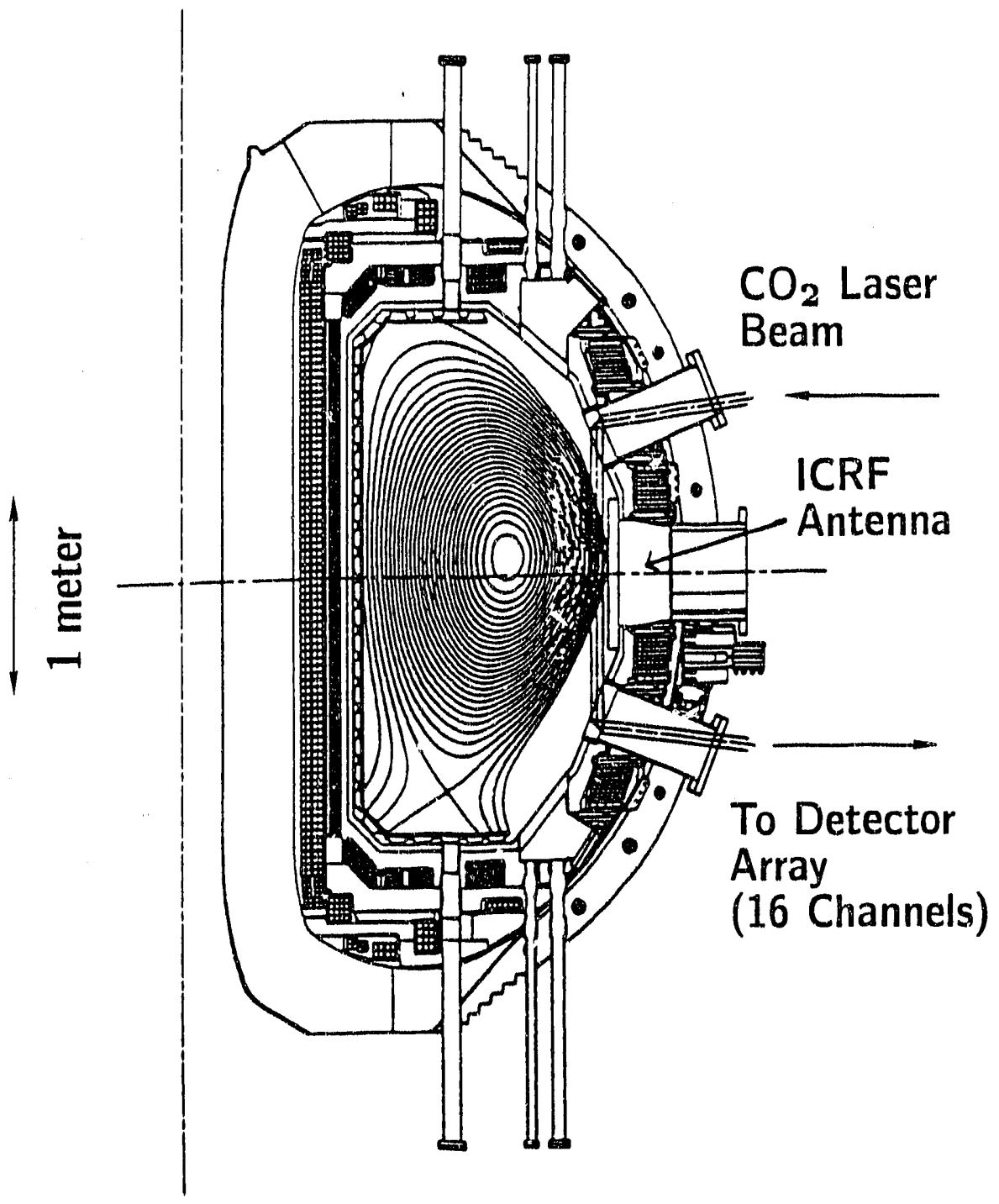

Fig. 1. Location of diagnostic on DIII-D.

where $\lambda_{0}=10.6 \mu \mathrm{m}, r_{\mathrm{e}}=2.8 \times 10^{-13} \mathrm{~cm}$ (classical electron radius), $z$ is the vertical direction of propagation and $R$ is the major radius. It can be shown that the radial fluctuation wavenumber must not exceed a given $k_{\max }$ in order for Eq. (1) to be valid. In our apparatus, a low-pass filter limits the spectrum to $k \leq 15 \mathrm{~cm}^{-1}$.

The plasma acts as a phase object, and no amplitude variation will be recorded by an image-forming instrument. Non-imaging techniques, such as scintillation ${ }^{13}$ and far-forward scattering, ${ }^{14}$ have been employed to measure plasma density fluctuations; 
these methods offer the advantage of simplicity but display an undesirably nonuniform response in $k$-space which renders data interpretation difficult. Far-field measurements such as conventional scattering ${ }^{4,5}$ resolve the individual $k$ components from scaitering volumes which increase in size for decreasing wavenumbers, thereby losing spatial resolution.

Imaging techniques which involve manipulation of the beam to transform the phase shift into a measurable irtensity variation offer in general a more regular $k$-space response. Of these methods, those involving interference with an external reference beam are vulnerable to mechanical vibrations and subsequent loss of sensitivity. Techniques that rely on an internal reference have the inherent limitation of not being able to resolve fluctuation wavelengths longer than the beam width. Among the several such methods available (including Schlieren and dark ground) phase contrast is the only one that offers true "phase imaging," i.e., the signal at the image plane is proportional to the phase at the corresponding point in the plasma (this is true in the approximation $|\Delta \Phi| \ll 1$, which is generally a very good one in the case of fluctuations above $1 \mathrm{kHz}$ ). The asymmetry between positive and negative orders in all the Schlieren techniques has the effect of summing different wavenumber components with different weights, thus rendering image formation impossible.

The phase contrast technique is based on the observation that to first order in $\Delta \Phi$, the transmitted wave consists of the original incident plane wave plus a small localized disturbance which carries all the information about the plasma structure, and which lags in phase by $90^{\circ}$. This can be seen mathematically by expanding the wave field $\mathrm{E}(R, t)=\mathrm{E}_{0} \mathrm{e}^{-i \omega_{0} t+i \Delta \Phi(R, t)}$ into

$$
\mathrm{E}(R, t) \simeq \mathrm{E}_{0} \mathrm{e}^{-i \omega_{0} t}[1+i \Delta \Phi(R, t)]
$$

The first term in the square brackets is the direct beam, while the second term is the diffracted beam. ${ }^{15}$ The two components can be separated physically in the focal plane of a lens. If a spatial filter is introduced that can shift the phase of the direct beam by $90^{\circ}$, while also reducing its intensity by a factor $\rho$, the two beam components 
will then interfere constructively or destructively in the image plane, with a resulting intensity

$$
\mathrm{I}(x, t) \simeq\left|\mathrm{E}_{0}\right|^{2}\left[\rho \pm 2 \sqrt{\rho} \Delta \Phi\left(\frac{x}{M}, t\right)\right]
$$

where $x$ is the coordinate of the radial image, and $M$ is the magnification. The time-dependert component of the signal is now proportional to the original phase shift, thus, according to Eq. (1), to the line integral of the density perturbation. The vertical integration implies that no spatial resolution can be achieved along the vertical direction (the effective integration length is typically $60 \mathrm{~cm}$ in our case).

The actual experiment is performed with a gaussian beam truncated by an aperture stop at the $\mathrm{e}^{-2}$ width $(7.6 \mathrm{~cm}$ ). A focal filter (phase mirror) is made by applying a $1.3 \mu \mathrm{m}\left(=\lambda_{0} / 8\right)$ thick reflective coating on a blank substrate with a surface reflectivity of $\rho$, except for a central band which is left uncoated. The width of the band is of the order of the focal spot size, and it corresponds to a given cutoff wavenumber $k_{\mathrm{c}}$.

The wavenumber response function can be calculated analytically in the approximation of spatial invariance:

$$
T(k)=\frac{2}{\pi} \mathrm{I}_{0} \sqrt{\rho} \int_{-1}^{1} \mathrm{e}^{-y^{2}} \frac{\sin \left(k_{c} w y\right)}{y}[1-\cos (k w y)] d y
$$

where $I_{0}$ is the beam intensity and $w$ is the gaussian half-width. This function is plotted in Fig. 2. For a sinusoidal phase shift $\Delta \phi(R, t)=\phi_{0} \cos \left(k R-w t+\psi_{0}\right)$, the ac signal intensity at the detector will be $I_{B C}(x, t)=\mathrm{T}(k) \Delta \phi(x / M, t)$.

The sensitivity of the measurement is determined by detector noise, which is predominantly shot noise in a photon detector. The signal to noise ratio is

$$
\mathrm{S} / \mathrm{N}=\frac{2 A \eta \mathrm{I}_{0}}{\hbar \omega_{0}} \frac{(\Delta \Phi)^{2}}{\Delta f}
$$

where $A$ is the element area, $\eta$ the quantum efficiency, and $\Delta f$ the bandwidth. Although the $\mathrm{S} / \mathrm{N}$ ratio does not depend directly on $\rho$, using a phase mirror with $\rho<1$ allows larger values of $\mathrm{I}_{0}$ to be attained without reaching saturation. In practice, it is desirable to operate at the highest possible power levels. 


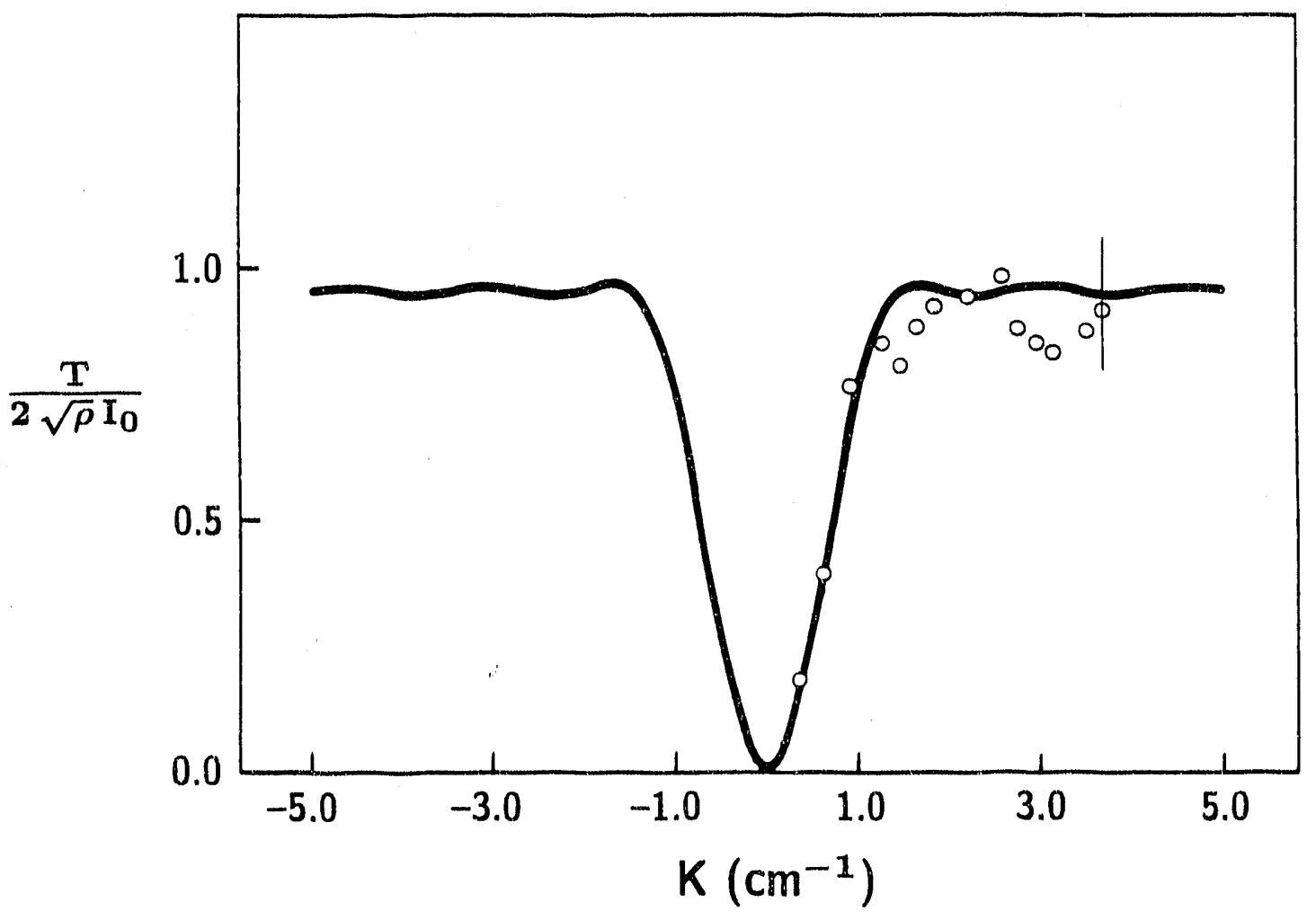

Fig. 2. Phase contrast response function (curve is calculated, circles represent experimental measurements from sound waves).

\section{EXPERIMENTAL APPARATUS}

The light source is a $20 \mathrm{~W} \mathrm{CO}_{2}$ laser. A continuously variable $\mathrm{ZnSe}$ attenuator is available to reduce the power below detector saturation. The gaussian beam is expanded to a width of $7.6 \mathrm{~cm}$ by a telescope composed of a $\mathrm{ZnSe}$ lens and an offaxis parabolic mirror. All these components are mounted on an optical table as are the imaging optics. Eight large mirrors relay the beam to and from the DIII-D vessel, which is accessed through two $\mathrm{ZnSe}$ windows. At the focus of a second off-axis parabolic mirror $(F=203 \mathrm{~cm}$ ) is the phase mirror. An image of the plasma is created on the plane of the detector by the parabolic mirror combined with iwo additional ZnSe lenses. The setup is flexible and allows variable magnification (to reduce the range and increase the spatial resolution) as well as image orientation. 
The long optical path, the lack of isolation of the several mirrors mounted on, and around the vessel, and mechanical vibrations during plasma discharges cause large movements in the focal spot position. In order to keep the focal spot centered on the phase-shifting groove, a fast, high-gain, dual-axis feedback stabilization system is used, including two small rotating mirrors located before the phase mirror. The position of the spot is measured by a quadrant, $\mathrm{LN}_{2}$ cooled, $\mathrm{HgCdTe}$ detector.

Phase mirrors of different sizes and materials were employed in the testing phase. The width that optimized the response function was found to be $480 \mu \mathrm{m}$. The data described in the present work were collected with a mirror of that size, made of Alcoated steel, lent to us by the TCA team. More recently, we started testing three new mirrors with grooves of the same size, with $\mathrm{Ge}, \mathrm{ZnSe}$, and $\mathrm{BaF}_{2}$ substrates $(\rho=0.36$, 0.17 , and 0.03 , respectively).

The detector is a 16 -element, $\mathrm{LN}_{2}$-cooled, photovoltaic $\mathrm{HgMnTe}$ linear array, followed by 16 low-noise preamplifiers, operating in the bandwidth $1 \mathrm{kHz}-100 \mathrm{MHz}$. The signals can either be digitized directly (after a series of filtering and amplification stages) at a $2 \mathrm{MHz}$ sampling rate for investigating broad-band turbulence, or they can be heterodyned with an rf reference signal during ICRF studies.

\section{TESTS AND FIRST PLASMẢ DATA}

The Phase Contrast Interferometer can be tested on acoustic and ultrasonic waves propagating in air in a direction perpendicular to the laser beam. The index of refraction of air depends linearly on the pressure perturbation. A simple test apparatus, consisting of a loudspeaker and a calibrated microphone, was assembled to measure the wavenumber response and to absolutely calibrate the diagnostic. Figure 2 shows the measured response function along with the theoretical function.

The diagnostic has been used only recently to study broadband density turbulence on the DIII-D tokamak $(R=1.67 \mathrm{~m}, a=67 \mathrm{~cm})$ in both L-mode and $\mathrm{H}$-mode plasmas. The most direct way to explore the spatial structure of dominant modes is to measure the two-point correlation function. Figure 3 shows the real part of the correlation function for a highly coherent mode observed at $96 \mathrm{kHz}$ during $\mathrm{H}$-mode. The eight points shown correspond to the innermost half of the beam, with the separatrix being approximately in the middle. The spatial Fourier transform peaks at $k_{R}=1.4 \mathrm{~cm}^{-1}$, corresponding to the sinusoidal waveform shown in 


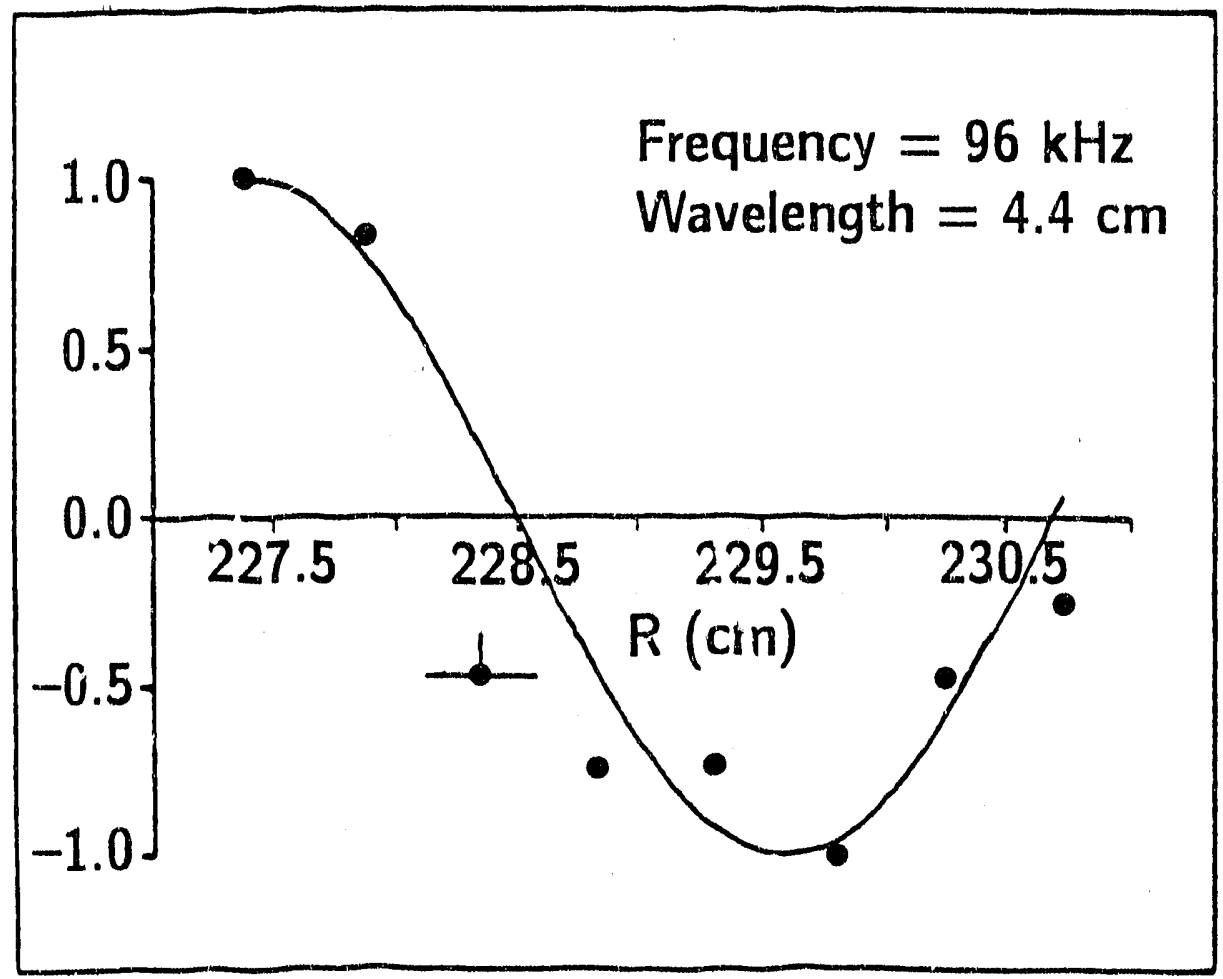

Fig. 3. Two-point correlation function of density fluctuations, at given frequency and at given time during $H$-mode $\left(I_{p}=1 M A, B_{T}=\right.$ 2.1 T). The wave shown corresponds to the peak of the spatial Fourier transform.

the figure. Figure 4 shows the time evolution of the auto-correlation spectrum for the same discharge.

Although correlation methods can provide valuable information, the diagnostic can also resolve rapidly moving structures unrevealed by correlation functions. This powerful capability is unique to the phase contrast technique, owing to its property of preserving the temporal and spatial form of the density fluctuations. We plan to use this technique in the coming year to carry out detailed measurements on low frequency turbulence in the plasma edge in DIII-D.

\section{ACKNOWLEDGMENTS}

The authors would like to thank the DIII-D RF group for their support during this work. We would also like to acknowledge valuable discussions with Dr. H. Weisen 


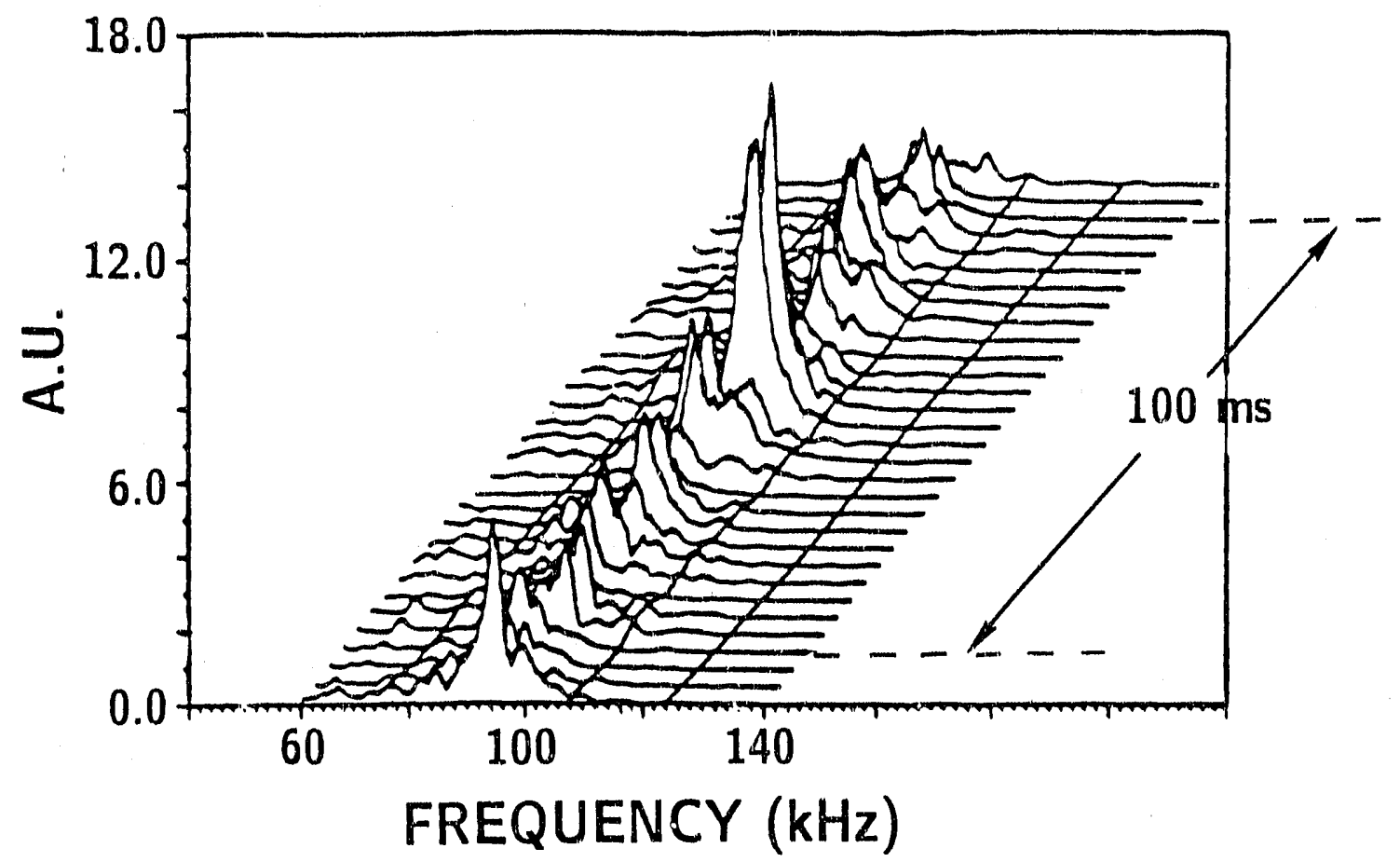

Fig. 4. Time evolution of auto-correlation spectrum of fluctuations at given spatial location.

of the CRPP in Lausanne and, in particular, we thank him for the loan of several phase mirrors. This work was supported by the U.S. Department of Energy under MIT Grant No. DE-FG02-91ER54109, and General Atomics Contract No. DE-AC0389ER.51114, and by the Consiglio Nazionale delle Ricerche (Italy).

\section{REFERENCES}

1 R.J. Fonck, R. Durst, Y.J. Kim, S.F. Paul, N. Bretz, R. Nazikian, Bull. Am. Phys. Soc. 36, 2445 (1991).

2 H. Biglari, P.H. Diamond, M.N. Rosenbluth, Phys. Fluids B 1, 109 (1989).

3 V.S. Chan, D.M. Meade, APS News, Vol. 1, 3, 64 (1992).

4 R.E. Slusher, C.M. Surko, Phys. Fluids 23, 472 (1980). 
5 R. Philipona, E.J. Doyle, N.C. Luhmann, Jr., W.A. Peebles, C. Rettig, K.H. Burrell, R.J. Groebner, H. Matsumoto, and the DIII-D Group, Rev. Sci. Instrum. 61, 3007 (1990).

๑ A.E. Costley, P. Cripwell, R. Prentice, A.C.C. Sips, Rev. Sci. Instrum. 61, 2823 (1990).

7 M.J. Mayberry, S.C. Chiu, M. Porkolab, V. Chan, R. Freeman, R. Harvey, R. Pinsker, "60 MHz Fast Wave Current Drive Experiment for DIII-D," in Radio-Frequency Power in Plasmas (Proc. 8th Top. Conf. Irvine, 1989) No. 190, AIP, New York (1989) 298.

8 M. Porkolab, "Parametric Instabilities in the Tokamak Edge Plasma in the Ion Cyclotron Heating Regimes," Fusion Engineering and Design 12, 93 (1990).

${ }^{9}$ F. Zernicke, Physica 9, 686, 974 (1942).

10 H.M. Presby, D. Finkelstein, Rev. Sci. Instrum. 38, 1563 (1967).

11 H. Weisen, Rev. Sci. Instrum. 59, 1544 (1988).

12 F.F. Chen, Introduction to Plasma Physics and Controlled Fusion, Plenum Press, New York, 1984.

13 L.E. Sharp, Plasma Phys. 25, 781 (1983).

14 D.E. Evans, E.J. Doyle, D. Frigione, M. von Hellermann, A. Murdoch, Plasma Phys. 25, 617 (1983).

15 E. Hecht, Optics, Addison-Wesley, Reading, 1987. 

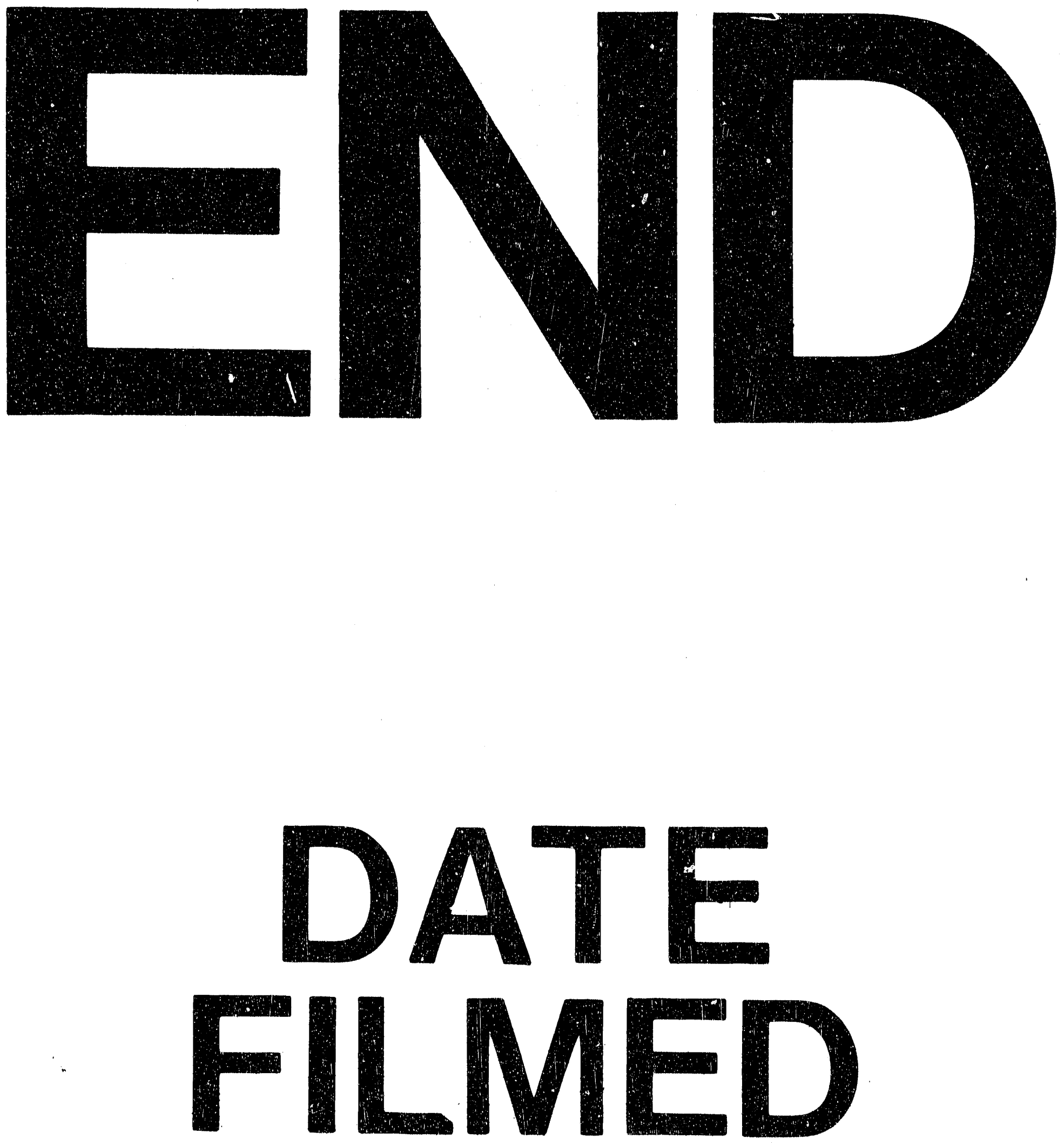

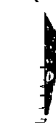

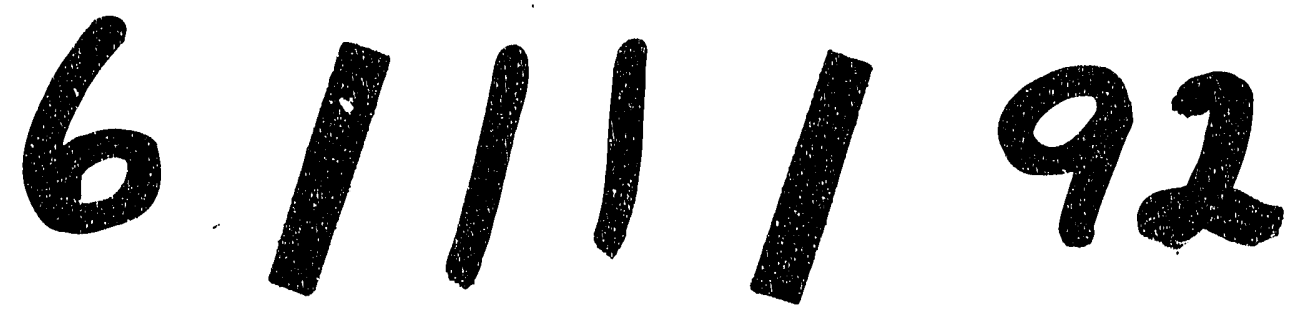


